\title{
A maximum entropy approach for the evaluation of surrogate endpoints based on causal inference.
}

\author{
Ariel Alonso $^{1, *}$, Wim Van der Elst ${ }^{2}$ \& Geert Molenberghs ${ }^{1,3}$
}

The maximum entropy principle offers a constructive criterion for setting up probability distributions on the basis of partial knowledge. In the present work, the principle is applied to tackle an important problem in the surrogate marker field, namely, the evaluation of a binary outcome as a putative surrogate for a binary true endpoint within a causal inference framework. In a first a step, the maximum entropy principle is used to determine the relative frequencies associated with the values of the vector of potential outcomes. Subsequently, in a second step, these relative frequencies are used in combination with two newly proposed metrics of surrogacy, the so-called individual causal association and the surrogate predictive function, to assess the validity of the surrogate. The procedure is conceptually similar to the use of non-informative or reference priors in Bayesian statistics. Additionally, approximate, identifiable bounds are proposed for the estimands of interest and their performance is studied via simulations. The methods are illustrated using data from a clinical trial involving schizophrenic patients and a newly developed and user friendly $R$ package Surrogate is provided to carry out the validation exercise. Copyright (c) 2015 John Wiley \& Sons, Ltd.

Keywords: Causal inference, Information theory, Maximum entropy, Surrogate endpoints.

\section{Introduction}

Joffe and Greene [1] studied several evaluation methods for surrogate endpoints and classified them into the socalled causal effects (CE) and causal association (CA) frameworks. In the former, the causal treatment effect on the true endpoint is assessed when the candidate surrogate is fixed at different values, whereas in the latter the association between the causal treatment effects on the surrogate and true endpoint is the focus of the analysis. In the CE framework, Gilbert and Hudgens [2] considered categorical and continuous outcomes as surrogates for a binary true endpoint and introduced the so-called causal effect predictiveness surface to evaluate the predictive value of a principal surrogate. Furthermore, within a CA framework, Li et al. [3] considered the setting in which the

${ }^{1}$ I-BioStat, KU Leuven, B-3000 Leuven, Belgium.

2 Janssen Pharmaceutical Companies of Johnson \& Johnson, Turnhoutseweg 30, B-2340 Beerse, Belgium.

${ }^{3}$ I-BioStat, Universiteit Hasselt, B-3500 Hasselt, Belgium.

*Correspondence to: Ariel Alonso, Kapucijnenvoer 35 blokd (bus 7001), 3000 Leuven, Belgium. Email: ariel.alonsoabad@kuleuven.be 


\section{Statistics}

\section{in Medicine}

surrogate was also binary and proposed a Bayesian modeling approach to estimate the parameters of interest under the assumption of monotonicity. Working with binary endpoints in a CA framework, Alonso et al. [4] introduced the individual casual association (ICA), an information-theoretic metric of surrogacy with a direct interpretation in terms of prediction accuracy. In addition, within a CE framework, Alonso et al. [5] introduced the so-called surrogate predictive function (SPF) to assess the validity of a binary surrogate for a binary true endpoint.

One of the major challenges faced when using causal inference models for the evaluation of surrogate endpoints, is the unidentifiability of the estimands of interest. This type of problem is often tackled in the literature by defining identifiability assumptions. Identifiability conditions are frequently combined with additional modeling assumptions in order to point identify the parameters of interest. For instance, Li et al. [3] treated the unobserved potential outcomes as missing variables and applied imputation methods. Identifiability was achieved under the assumption of monotonicity by selecting appropriate prior distributions for the unidentifiable parameters. In order to achieve identification of the causal effect predictiveness surface, Gilbert and Hudgens [2] focused on the setting where the surrogate under the control was fixed and known and assumed that the value of the surrogate potential outcome for the new treatment $S_{1}$ in the control group, could be predicted using baseline covariates.

These identifiability assumptions are mostly untestable from the data and, consequently, none of them will always deliver unbiased results. Therefore, the availability of several identifiability methods is desirable, as all them together will comprise a sensitivity analysis that will allow researchers to assess how the main conclusions vary with varying assumptions. In the present work, we propose an information-theoretic approach to address the nonidentifiability problem, based on the maximum entropy principle (MEP). The MEP states that, when drawing inferences based on incomplete information, one should use the probability distribution with maximum entropy permitted by the data at hand. The antecedents of the principle and its rationale can be found in statistical mechanics and the second law of thermodynamics. The MEP has been successfully applied to derive non-informative priors in Bayesian analysis, the so-called reference priors [6, 7]. In the present context, its application leads to the assessment of the surrogate based on the maximum entropy distribution of the vector of potential outcomes. Basically, the maximum entropy distribution is the one that most closely approximates equal probabilities for all 16 cells of the joint distribution of the potential outcomes in the appropriate metric, subject to the constraints inherent in the data. If the constraints from the observed data are not taken into account, then the maximum entropy distribution would apply a mass of $1 / 16$ to all 16 cells of the joint distribution. The MEP approach proposed in the following sections is conceptually similar to the use of non-informative or reference priors in Bayesian statistics, in the sense that it gives the most general/non-informative assessment of the parameters of interest, namely, the ICA and SPF. Jaynes [8] justified the use of the MEP in the following terms: The only way known to set up a probability distribution that fairly represents a state of incomplete knowledge is to maximize the entropy, subject to all the information we have. Any other distribution would necessarily either assume information that we do not have, or contradict information that we do have.

In addition, providing identifiable bounds for the estimands of interest, as proposed by Gadbury, Iyer and Jeffrey [9], is another useful strategy. Building upon the work of Alonso et al. [4] we propose approximate, identifiable bounds for the ICA and SPF and a simulation study was carried out to assess the performance of these bounds.

In Section 2, the causal-inference model underlying the evaluation strategy is introduced. In Section 3, two evaluation metrics, the so-called individual causal association and the surrogate predictive function, are reviewed. The MEP approach is introduced in Section 4 and its relationship with maximum likelihood (ML) estimation is discussed in Section 5. Building on the work presented in Alonso et al. [4], new approximate, identifiable bounds for the ICA and SPF are proposed in Section 6 and a simulation study is carried out to assess their performance. The case study is presented and analyzed in Section 7 and some final comments are given in Section 8. A list of the most frequently used abbreviations can be found in Table 1 . 


\section{Causal inference model}

In the rest of the manuscript it will be assumed that data on both the true $(T)$ and surrogate $(S)$ endpoint are available from a single clinical trial and that only two treatments are under evaluation $(Z=0 / 1)$. Further, it will be assumed that both endpoints are binary variables coded as 1 when a beneficial outcome is observed and 0 otherwise. In addition, the standard stable unit treatment value assumption (SUTVA) will also be made [10].

The evaluation exercise will be based on the so-called Rubin's model for causal inference. This model assumes the existence of a four-dimensional vector of potential outcomes $\boldsymbol{Y}=\left(T_{0}, T_{1}, S_{0}, S_{1}\right)^{\prime}$ for each patient. The components $T_{1}, S_{1}, T_{0}$ and $S_{0}$ represent the outcomes for the true and surrogate endpoint of an individual had he received the treatment or control, respectively. On account of simplicity, in the following the discussion will be temporarily restricted to the surrogate endpoint, but similar arguments can be put forward for the true endpoint as well.

The parameters $\pi_{i j}^{S}=P\left(S_{0}=i, S_{1}=j\right)$ with $i, j=0,1$, fully characterize the bivariate distribution of the vector of potential outcomes for the surrogate $\boldsymbol{Y}_{S}=\left(S_{0}, S_{1}\right)^{\prime}$ and this parametrization leads to the marginals $\pi_{i .}^{S}=\sum_{j} \pi_{i j}^{S}, \pi_{. j}^{S}=\sum_{i} \pi_{i j}^{S}$. However, most often only one of the two potential outcomes $S_{0}$ and $S_{1}$ can be observed and the distribution of $\boldsymbol{Y}_{S}$ is therefore not identifiable [11]. In particular, the association structure of the two potential outcomes cannot be inferred from the data. Unlike the association structure, the marginal probabilities $\pi_{S}=\left(\pi_{0}^{S}, \pi_{1}^{S}, \pi_{\cdot 0}^{S}, \pi_{\cdot 1}^{S}\right)^{\prime}$ are identifiable under fairly general conditions. Indeed, under SUTVA, $S=Z S_{1}+(1-Z) S_{0}$ and if the treatment assignment mechanism is independent of the potential outcomes $\left(\boldsymbol{Y}_{S} \perp Z\right)$, then $\pi_{1 \cdot}^{S}=E(S \mid Z=0)$ with $\pi_{0}^{S}=1-\pi_{1}^{S}$ and $\pi_{\cdot 1}^{S}=E(S \mid Z=1)$ with $\pi_{\cdot 0}^{S}=1-\pi_{\cdot 1}^{S}$. Notice that, due to the random treatment allocation, the aforementioned assumption of independence $\boldsymbol{Y}_{S} \perp Z$ can often be guaranteed in randomized clinical trials.

As previously stated, in order to identify the entire bivariate distribution of $\boldsymbol{Y}_{S}$ additional assumptions on the association structure are needed. To this end, let us now consider the odds ratio $\theta_{S}=\pi_{00}^{S} \pi_{11}^{S} / \pi_{10}^{S} \pi_{01}^{S}$. Using $\theta_{S}$ and the marginal probabilities, the full bivariate distribution of $\boldsymbol{Y}_{S}$ can be recovered [12].

The individual causal effect of the treatment on the surrogate can be defined as $\Delta S=S_{1}-S_{0}$; it follows a multinomial distribution with parameters $\pi_{i}^{\Delta S}=P(\Delta S=i)=\sum_{p q} \pi_{p q}^{S}, i=-1,0,1$, where the sum is taken over all sub-indexes $p, q$ satisfying $q-p=i$. Although the distribution of $\Delta S$ is not generally identifiable from the data, once $\theta_{S}$ is fixed, it becomes fully identifiable.

Like before, the potential outcomes $\boldsymbol{Y}_{T}=\left(T_{0}, T_{1}\right)^{\prime}$ can be used to define the individual causal treatment effect on the true endpoint $\Delta T$ and its distribution. The vector of individual causal treatment effects $\Delta=(\Delta T, \Delta S)^{\prime}$, which follows the multinomial distribution given in Table 2, is the fundamental quantity used in the following sections to assess the validity of the surrogate.

[Insert Table 2 about here]

\section{Individual causal association: An information-theoretic approach}

Shannon [13] introduced the concept of entropy to quantify the "epistemic" uncertainty or lack of knowledge implied by a distribution. If $Y$ is a discrete random variable taking values $\left\{y_{1}, y_{2}, \ldots, y_{m}\right\}$ with probability function $P\left(Y=y_{i}\right)=\pi_{i}$, then the entropy of $Y$ is given by $H(Y)=-E_{Y}[\log P(Y)]=-\sum_{i} \pi_{i} \log \pi_{i}$.

Similarly, the joint and conditional entropies are defined as $H(X, Y)=-E_{X, Y}[\log P(X, Y)]$ and $H(Y \mid X)=$ $-E_{X}\left[E_{Y}(\log P(Y \mid X))\right]$, with $P(x, y)$ and $P(y \mid x)$ denoting the joint and conditional probability functions, respectively. Entropy is always non-negative and satisfies $H(Y \mid X) \leq H(Y)$ for any pair of random variables 


\section{Statistics}

\section{in Medicine}

$(X, Y)$, with equality holding under independence. The foregoing inequality states that, as an average, uncertainty on $Y$ can only decrease if additional information $(X)$ becomes available. Furthermore, entropy is invariant under a bijective transformation.

The amount of uncertainty in $Y$, expected to be removed if the value of $X$ were known, is quantified by the so-called mutual information $I(X, Y)=H(Y)-H(Y \mid X)$. Mutual information is invariant under bijective transformations of $X$ and $Y$ and satisfies $I(X, Y) \geq 0$ with $I(X, Y)=0$ if and only if $X$ and $Y$ are independent. Moreover, $I(X, Y)=I(Y, X)$ and $I(X, X)=H(X)$. It follows from the definitions of entropy and mutual information that

$$
I(X, Y)=H(X)+H(Y)-H(X, Y)=\sum_{x, y} P(x, y) \log \left(\frac{P(x, y)}{P(x) P(y)}\right)
$$

where $P(x, y), P(x)$, and $P(y)$ denote the joint and marginal probability functions of $X$ and $Y$, respectively.

Understanding the association between the causal treatment effects on the true and surrogate endpoint is critical to understanding the value of a surrogate from a clinical perspective [14]. Along these lines, Alonso et al. [4, 15] proposed to assess surrogacy using the so-called individual causal association (ICA). When both endpoints are continuous and normally distributed these authors quantified the ICA using the Pearson correlation coefficient $\rho_{\Delta}=\operatorname{corr}(\Delta T, \Delta S)$ [15]. In addition, for binary endpoints, Alonso et al. [4] introduced the following informationtheoretic measure of association to assess the ICA:

$$
R_{H}^{2}(\Delta T, \Delta S)=\frac{I(\Delta T, \Delta S)}{\min [H(\Delta T), H(\Delta S)]},
$$

where the term in the numerator is defined as

$$
I(\Delta T, \Delta S)=\sum_{i, j=-1}^{1} \pi_{i j}^{\Delta} \log \left(\frac{\pi_{i j}^{\Delta}}{\pi_{i}^{\Delta T} \pi_{j}^{\Delta S}}\right),
$$

and the denominator equals the minimum of the entropies of the individual causal treatment effects defined as $H(\Delta T)=\sum_{i=-1}^{1} \pi_{i}^{\Delta T} \log \left(\pi_{i}^{\Delta T}\right)$ and $H(\Delta S)=\sum_{j=-1}^{1} \pi_{j}^{\Delta S} \log \left(\pi_{j}^{\Delta S}\right)$.

Using some theoretical considerations, Alonso et al. [4] showed that, at least in some scenarios, the ICA may offer a more coherent assessment of surrogacy than other previously introduced metrics.

The ICA, as given in (1), can also be interpreted as a measure of prediction accuracy, i.e., a measure of how accurately one can predict the causal treatment effect on the true endpoint for a given individual, using his causal treatment effect on the surrogate. Indeed, Alonso et al. [4] showed that $R_{H}^{2}(\Delta T, \Delta S)$ is invariant under one-to-one transformations and that it always lies in the unit interval, taking a zero value when $\Delta T$ and $\Delta S$ are independent and value one when there is a nontrivial transformation $\psi$ so that $P[\Delta T=\psi(\Delta S)]=1$. Consequently, when $R_{H}^{2}(\Delta T, \Delta S)=1$ there exists a deterministic relationship between both individual causal treatment effects, namely $\Delta T=\psi(\Delta S)$, and $\Delta S$ predicts $\Delta T$ without error. In addition, when $R_{H}^{2}(\Delta T, \Delta S)=0$ both individual causal treatment effects are independent and no meaningful predictions are possible.

Even though $R_{H}^{2}(\Delta T, \Delta S)$ does provide a quantification of the surrogate predictive value, it does not give any information regarding the specific form of the prediction function $\psi$ and leaves some important scientific questions unanswered. For instance, it does not allow to assess how likely it is that the treatment will have a negative impact on the true endpoint, given that it has a beneficial effect on the surrogate, i.e., the probability that the surrogate will produce a false positive result.

To solve these problems, Alonso et al. [5] introduced the so-called surrogate predictive function (SPF) defined as $r(i, j)=P(\Delta T=i \mid \Delta S=j)$. The SPF fully captures the relationship between the individual causal treatment 
effect on the surrogate and true endpoint, and it allows to address some important scientific questions. For example, $r(-1,1)$ quantifies the probability that the surrogate will produce a false positive result. The SPF is also related to concepts previously introduced in the literature, for instance, it is intrinsically related to the concept of causal necessity proposed by Frangakis and Rubin [16]. These authors defined that $S$ is necessary for the effect of treatment on the outcome $T$, if a causal effect of treatment on $T$ can occur only if a causal effect of treatment on $S$ has occurred. Essentially, causal necessity can be re-stated as $r(0,0)=1$.

Furthermore, there is a close relationship between $r(i, j)$ and the best prediction function associated with the distribution of $\Delta$. To illustrate this let us first define the best prediction function as the function $\psi_{b}=$ $\arg \max _{\psi} P[\Delta T=\psi(\Delta S)]$. Alonso et al. [5] showed that $\psi_{b}$ can be written as a function of $r(i, j)$. Obviously, if $R_{H}^{2}=1$ then $P\left[\Delta T=\psi_{b}(\Delta S)\right]=1$.

\section{Identifiability issues: A maximum entropy approach}

The ICA and SPF are both based on the distribution of the vector of individual causal treatment effects $\Delta^{\prime}=(\Delta T, \Delta S)$, given in Table 2. However, this distribution is not identifiable from the data and, therefore, neither the ICA nor the SPF can be directly estimated.

In the present work, we propose to tackle this problem using the MEP and based on the distribution of the vector of potential outcomes. To this end, let us denote by $N$ the total number of patients included in the study and by $\boldsymbol{Y}_{1}, \boldsymbol{Y}_{2}, \ldots, \boldsymbol{Y}_{N}$ their corresponding vectors of potential outcomes. Further, let us define the outcome vector $\boldsymbol{n}=\left(n_{i j p q}\right)$ where $n_{i j p q}$ is the number of patients with $\boldsymbol{Y}=(i, j, p, q)$ and $i, j, p, q=0 / 1$. If $\boldsymbol{\pi}=\left(\pi_{i j p q}\right)$, with multinomial probabilities $\pi_{i j p q}=P\left(T_{0}=i, T_{1}=j, S_{0}=p, S_{1}=q\right)$, then the probability of any given outcome vector $\boldsymbol{n}$ is given by

$$
P(\boldsymbol{n} \mid \boldsymbol{\pi})=W(N, \boldsymbol{n}) \prod_{i j p q} \pi_{i j p q}^{n_{i j p q}}=W(N, \boldsymbol{n}) G(\boldsymbol{n} \mid \boldsymbol{\pi}),
$$

where

$$
W(N, \boldsymbol{n})=\frac{N !}{\prod_{i j p q} n_{i j p q} !} .
$$

The multinomial coefficient $W(N, \boldsymbol{n})$ (a.k.a. the multiplicity term) quantifies the number of ways in which a specific outcome vector $\boldsymbol{n}$ can be realized. In addition, the probability of observing a particular sequence of responses $\boldsymbol{Y}_{1}, \boldsymbol{Y}_{2}, \ldots, \boldsymbol{Y}_{N}$, producing the outcome vector $\boldsymbol{n}$, is given by $G(\boldsymbol{n} \mid \boldsymbol{\pi})$. Using this notation, one can define the vector of relative frequencies $\boldsymbol{p}=\left(p_{i j p q}\right)$ where $p_{i j p q}=n_{i j p q} / N$. Although $\boldsymbol{p}$ is not observable, some functions of it are completely determined by the data. Indeed, the data at hand allow estimating three probabilities $P(T=t, S=s \mid Z)$ within each treatment group and, hence,

$$
\begin{aligned}
p_{1 \cdot 1 \cdot}=\hat{P}(T=1, S=1 \mid Z=0), & p_{\cdot 1 \cdot 1}=\hat{P}(T=1, S=1 \mid Z=1), \\
p_{1 \cdot 0 \cdot}=\hat{P}(T=1, S=0 \mid Z=0), & p_{\cdot 1 \cdot 0}=\hat{P}(T=1, S=0 \mid Z=1), \\
p_{0 \cdot 1 \cdot}=\hat{P}(T=0, S=1 \mid Z=0), & p_{\cdot 0 \cdot 1}=\hat{P}(T=0, S=1 \mid Z=1), \\
p_{\ldots .}=1, &
\end{aligned}
$$

with the points in the sub-indexes indicating sums of the components of $\boldsymbol{p}$ over those specific sub-indexes. If one further defines the vector $\boldsymbol{c}^{\prime}=\left(1, p_{1 \cdot 1 \cdot}, p_{1 \cdot 0 .}, p_{\cdot 1 \cdot 1}, p_{\cdot 1 \cdot 0}, p_{0 \cdot 1 \cdot,}, p_{\cdot 0 \cdot 1}\right)$, then all the identified restrictions given in (3) can be written as a system of linear equations $\mathbf{A} \boldsymbol{p}=\boldsymbol{c}$, with $\mathbf{A}$ a binary matrix (details provided in the Supplementary Materials Part A). The previous system of linear equations defines the class $\Omega_{C}$ of all relative 


\section{Statistics}

\section{in Medicine}

frequencies $\boldsymbol{p}$ compatible with the data at hand, i.e., $\Omega_{C}=\left\{\boldsymbol{p} \in \Re_{+}^{16}: \mathbf{A} \boldsymbol{p}=\boldsymbol{c}\right\}$. Essentially, the data $\boldsymbol{c}$ tells us that the actual vector $\boldsymbol{p}$ must have been in the class $\Omega_{C}$ but do not fully determine it.

Using Stirling's formula, the multiplicity term in (2) can be approximated, for large $N$, by $W(N, \boldsymbol{n}) \approx e^{N H(\boldsymbol{p})}$. The MEP instructs to use $\boldsymbol{p}^{*}=\arg \max \boldsymbol{p} \in \Omega_{C} H(\boldsymbol{p})$ as the basis for analysis. It is important to point out that the maximum entropy distribution produces the same association between the true and surrogate endpoints as the observable data in both treatment groups. This is so because the parameters governing the association between the surrogate and true endpoint are identifiable and, hence, "fixed" in the optimization procedure through the set of linear restrictions used to define $\Omega_{C}$.

Additional restrictions could also be considered when calculating the maximum entropy distribution. For instance, adding the equations $p_{10 . .}=0$ and $p_{* 10}=0$ to the system of linear equations (3), would allow to compute the maximum entropy distribution under the assumption of monotonicity for both endpoints. Although theoretically possible, adding untestable restrictions to $\Omega_{C}$ in some sense contradicts the essence of the MEP, which is to use the least informative distribution permitted by the data at hand. This is, however, a general principle and in particular applications users of the methodology may want to consider untestable but plausible restrictions when maximizing $H(\boldsymbol{p})$.

As previously stated, the concept of entropy quantifies the "epistemic" uncertainty or lack of knowledge implied by a distribution and, therefore, maximizing entropy minimizes the amount of prior information built into a distribution. In addition, there is an asymptotic combinatorial result that justifies further the use of the maximum entropy distribution. It is the so-called Entropy Concentration Theorem (ECT) [17]. The theorem basically states that if $N$ (number of patients included in the trial) is large enough, then $100(1-F) \%$ of all $\boldsymbol{p} \in \Omega_{C}$ will satisfy $H_{\max }-\Delta H \leq H(\boldsymbol{p}) \leq H_{\max }$, where $H_{\max }$ is the maximum entropy achieved by the maximum entropy distribution $\boldsymbol{p}^{*}$ and $\Delta H$ is a function of the upper tail area $1-F$ of the $\chi_{k}^{2}$ distribution, with $k=q-m-1$ degrees of freedom, $q$ denoting the dimension of $\boldsymbol{p}$, and $m$ denoting the dimension of $\Omega_{C}$ (the number of independent linear restrictions). Note that, in the surrogate evaluation context, $q=16$ and $m=7$.

In spite of all the previous theoretical developments, it is important to point out that the distribution of the vector of potential outcomes $\boldsymbol{Y}$ is unidentifiable and, consequently, it is impossible to empirically assess how frequently the true distribution of $\boldsymbol{Y}$ would be close to the maximum entropy distribution. In addition, justifying the use of the maximum entropy distribution in complex settings involving living organism based on physical laws, like the second law of thermodynamics, is conceptually problematic. That is the reason why the MEP approach should be used in a sensitivity analysis context and not as a stand alone procedure. As stated in the introduction, the use of the MEP is conceptually similar to the use of non-informative priors in Bayesian statistics. Indeed, often in Bayesian analysis, several prior distributions are used, in a sensitivity analysis context, to assess their impact on the posterior distribution. In such a setting, a non-informative prior is always used as a benchmark against which the impact of other more "subjective" priors can be evaluated. When evaluating surrogate endpoints, the MEP analysis can serve a similar purpose, which is, in our opinion, its fundamental additional value.

\subsection{Maximizing $H(\boldsymbol{p})$ on $\Omega_{C}$}

The entropy function $H(\boldsymbol{p})$ can be seen as a special case of the so-called Kullback-Leibler divergence between two distributions $\boldsymbol{p}$ and $\boldsymbol{q}$; given by $l(\boldsymbol{p} \mid \boldsymbol{q})=-\sum p_{k} \ln \frac{p_{k}}{q_{k}}$. Indeed, it is easy to see that if one takes $q_{i}=1 / 16$ then $l(\boldsymbol{p} \mid \boldsymbol{q})=-H(\boldsymbol{p})+\ln (16)$. Notice that, to simplify the notation, the components of $\boldsymbol{p}$ are now simply denoted by $p_{k}$ with $k=1,2, \ldots, 16$. The Kullback-Leibler divergence between $\boldsymbol{p}$ and $\boldsymbol{q}$ is a measure of the information lost when $\boldsymbol{q}$ is used to approximate $\boldsymbol{p}$. Brockett et al. [18] proved that $\max _{\boldsymbol{p} \in \Omega_{C}} H(\boldsymbol{p})$ is equivalent to

$$
\min _{\boldsymbol{\beta}} \nu(\boldsymbol{\beta})=-\frac{1}{16} \sum e^{\boldsymbol{a}_{i}^{\prime} \boldsymbol{\beta}-1}-\boldsymbol{c}^{\prime} \boldsymbol{\beta}
$$


where $\boldsymbol{a}_{i}$ denotes the $i$ th column of the matrix $\mathbf{A}$ and at their optimum values, $p_{i}^{*}=(1 / 16) e^{\boldsymbol{a}_{i}^{\prime} \boldsymbol{\beta}^{*}-1}$. Therefore, the maximum entropy relative frequency vector $\boldsymbol{p}^{*}$ can now easily be calculated as the optimal solution to an unconstrained dual convex programming problem, which involves only linear and exponential terms.

\subsection{Assessing ICA and SPF}

Once the maximum entropy relative frequency vector $\boldsymbol{p}^{*}$ has been determined, the distribution of the vector of individual causal effects $\Delta$, given in Table 2, can be estimated based on these values. Further, the individual causal association $R_{H}^{2}$ can be calculated using (1) and the SPF can be computed as $r(i, j)=\pi_{i j}^{\Delta} / \pi_{j}^{\Delta S}$ with $\pi_{j}^{\Delta S}=\sum_{j} \pi_{i j}^{\Delta}$.

\section{Maximum Likelihood versus MEP}

It looks somehow paradoxical that, if there is no information in the data to distinguish among a set of possible parameters based on the likelihood function, the maximum entropy (ME) approach does yield a single point estimate. In the following we offer some heuristic arguments that may help to clarify the differences between both approaches. The ML procedure maximizes expression (2) as a function of $\pi$, i.e.,

$$
\max _{\boldsymbol{\pi} \in Q} P(\boldsymbol{n} \mid \boldsymbol{\pi})=\max _{\boldsymbol{\pi} \in Q} W(N, \boldsymbol{n}) \prod_{i j p q} \pi_{i j p q}^{n_{i j p q}}
$$

with $Q=\left\{\boldsymbol{\pi} \geq \mathbf{0}, \mathbf{1}^{\prime} \boldsymbol{\pi}=\mathbf{1}\right\}$, or equivalently,

$$
\max _{\pi \in Q} \sum_{i j p q} p_{i j p q} \ln \pi_{i j p q}
$$

It can easily be shown that, after applying Lagrange multipliers, the previous maximization problem leads to the maximum likelihood estimates (MLE) $\hat{\pi}_{i j p q}=p_{i j p q}$. However, the proportions $p_{i j p q}$ are unobserved (only some margins are actually observable) and, consequently, the MLE cannot be uniquely determined. This is a direct consequence of the so-called fundamental problem of causal inference, i.e., the vector of potential outcomes $\boldsymbol{Y}=\left(T_{0}, T_{1}, S_{0}, S_{1}\right)^{\prime}$ is essentially unobservable and, therefore, the number of patients with $\boldsymbol{Y}=(i, j, p, q)$ and $i, j, p, q=0 / 1$, namely $n_{i j p q}$, cannot be determined either. Furthermore, it can be shown that the MLE $\hat{\pi}$ is the solution of the optimization problem

$$
\min _{\boldsymbol{\pi} \in Q} l(\boldsymbol{\pi} \mid \boldsymbol{p}),
$$

where $l(\boldsymbol{\pi} \mid \boldsymbol{p})$ is the Kullback-Leibler divergence between $\boldsymbol{\pi}$ and $\boldsymbol{p}$, i.e., the ML procedure selects the parameters closest, in the Kullback-Leibler divergence metric, to $\boldsymbol{p}$. As stated before, given that $\boldsymbol{p}$ is not observed, such an optimization problem does not have a unique solution. On the other hand, the ME procedure focuses on the following optimization problem

$$
\min _{\boldsymbol{p} \in \Omega_{C}} l\left(\boldsymbol{p} \mid \frac{1}{16} \mathbf{1}\right) .
$$

Notice that now the data are used only to define $\Omega_{C}=\left\{\boldsymbol{p} \in \Re_{+}^{16}: \mathbf{A} \boldsymbol{p}=\boldsymbol{c}\right\}$, i.e., the actual observed proportions $\boldsymbol{c}$ are used to define the restrictions in the optimization process. Any vector $\boldsymbol{p} \in \Omega_{C}$ will be compatible with the data at hand. From all those vectors, the procedure will select the one closest to the uniform distribution. In Bayesian probability, the uniform distribution is the simplest non-informative prior.

Intuitively, the ML procedure selects the values of the parameters that make the data most likely, whereas the ME selects those values compatible with the data at hand, that produce the distribution closest (in the Kullback-Leibler 


\section{Statistics}

\section{in Medicine}

divergence metric) to the most non-informative possible distribution, namely, the uniform distribution (if no further constraints are imposed, then the uniform distribution maximizes the entropy on $Q$ ). Given that the data required for the application of ML ( $\left.n_{i j p q}\right)$ is incomplete (only some margins are observed), the procedure fails to achieve identifiability. The ME procedure does not require the full data $\left(n_{i j p q}\right)$ but it only uses the observed margins $(c)$ to define the constrains for the optimization problem.

\section{Approximate bounds for the estimands of interest}

Alonso et al. introduced a two-step Monte Carlo procedure to assess the ICA and SPF [4, 5]. The procedure determines, in a first step, the region of the parametric space of the distribution of the potential outcomes that is compatible with the data at hand (the so-called $\Gamma_{D}$ region) and, subsequently, in a second step, a Monte Carlo approach is implemented to study the behavior of the ICA and SPF on this region. Essentially, in the Monte Carlo step, the values of the ICA and SPF are computed for several points uniformly sampled on $\Gamma_{D}$ and later summarized using frequency distributions and histograms. These frequency distributions and histograms describe the behavior of the ICA and SPF across all scenarios compatible with the data. Based on the previous algorithm, in the following, approximate identifiable bounds will be introduced for the ICA. Completely similar results can be obtained for the SPF as well.

Basically, one is interested in the behavior of the function ICA: $\Gamma_{D} \rightarrow[0,1]$. If, in the Monte Carlo step, the number of sampled $\pi$ vectors on $\Gamma_{D}$ is sufficiently large, then the observed $\left[\min _{\pi}\right.$ ICA, $\max _{\pi}$ ICA] should contain the true value of the ICA. Therefore, these minimum and maximum observed values could be interpreted as approximate bounds for the individual causal association.

\subsection{Simulation study}

We assessed the performance of the previous approximate bounds via simulations. It is important to point out that simulation studies have major limitations in this context. In fact, even though simulations are excellent tools to evaluate the performance of estimation and inferential procedures, their utility is limited when the parameters of interest are unidentifiable. Actually, the data do not convey enough information to estimate the parameters consistently and the same will be true for any generated data set. As stated before, untestable assumptions are often made to make inferences about the unidentifiable parameters. In such a situation it is always possible to design simulation studies where the proposed methodology produces biased results (the data generating mechanism does not satisfy the assumptions) or unbiased results (the data generating mechanism satisfies the assumptions). Neither the Monte Carlo procedure nor the MEP approach are designed to estimate the unidentifiable ICA, but to offer a sensitivity analysis about its plausible values. We argue that the MEP approach plays an especial role in this context because it delivers the conclusions derived from the most non-informative distribution for the vector of potential outcomes.

Two different scenarios were considered in the simulations: 1) Data generated under monotonicity and 2) Data generated under no monotonicity. In order to keep the length of the manuscript at a reasonable level, only a summary of the results for scenario (1) will be presented. The interested reader can find a complete discussion of the simulation study in the Supplementary Materials Part C.

Simulation setting 1: data generated under monotonicity A total of $5,000 \pi$ vectors were generated under the assumptions of monotonicity for $S$, monotonicity for $T$, and monotonicity for both $S$ and $T$. These 15,000 $\pi$ vectors, characterizing the distribution of the vector of potential outcomes $\boldsymbol{Y}$, represent the unknown "true 
stage of nature" and for each of them a data set containing $N=500$ patients was generated. These data sets were subsequently analyzed using the two-step Monte Carlo procedure.

When using the Monte Carlo procedure, for each generated data set, a total of 10,000 $\boldsymbol{\pi}$ vectors were uniformly sampled from those sub-regions of $\Gamma_{D}$ where monotonicity held for $S$, for $T$, for both $S$ and $T$ and from the subregion where monotonicity held neither for $S$ nor for $T$. Furthermore, the minimum and maximum observed values for the ICA were computed.

Results: Table 3 displays the setting where the data sets were generated assuming monotonicity for $S$ and $T$. Let us now discuss the results obtained in this setting, when the data were analyzed under the (wrong) assumption that monotonicity did not hold (top part of the table). Basically, for each generated data set, the Monte Carlo procedure was used to uniformly sample $10,000 \pi$ vectors from the sub-region $M_{N} \subset \Gamma_{D}$ where monotonicity did not hold. The percentage of cases (generated data sets) in which the true ICA was included in the [min, max] range of the ICA values (identified by the Monte Carlo procedure) is provided in the "\% True ICA included" column and the columns "Mean min(ICA)" and "Mean max(ICA)" show the average of the ICA minimum and maximum values obtained from the generated data sets.

Unsurprisingly, a large proportion of theses analyses led to a range [min, $\max ]$ that did not contain the true ICA value (see "\% True ICA included" column). Given that $M_{N}$ never contained the true $\boldsymbol{\pi}$ vector in this setting, it is not surprising that the bounds derived from it were largely misleading. The bottom part of Table 3 shows the results obtained when the data were analyzed under the (correct) assumption that monotonicity held for both endpoints. Thus, the Monte Carlo procedure was used to uniformly sample 10, $000 \boldsymbol{\pi}$ vectors from the sub-region $M_{S T} \subset \Gamma_{D}$ where monotonicity held for both $S$ and $T$. Notice that now the true ICA was contained in the [min, max] interval in almost all the analyses. Given that one is now exploring the behavior of the ICA in a region that always contained the true $\pi$ vector, i.e. the $M_{S T}$ region, this result comes as no surprise. Obviously, the true ICA value will also be contained in the $[\mathrm{min}, \max ]$ interval calculated after combining the results from all the analyses, i.e., the analyses carried out under all the monotonicity assumptions. However, such an interval will be, in general, wider than the one obtained under the correct monotonicity assumption. The other scenarios led to essentially the same findings and the details are provided in the Supplementary Materials Part C.

\section{Case Study}

The lack of user-friendly software is a challenge frequently encountered by data analysts when working with surrogate endpoints. The problem is compounded by the fact that the most sound approaches are oftentimes rather complex and difficult to implement. The $\mathrm{R}$ package Surrogate, freely available at http://cran.r-project.org/web/packages/Surrogate/, allows to carry out the proposed evaluation procedure in a rather straightforward fashion. For conciseness, in the present section only the main results are given and no reference to the software is made. However, in the supplementary materials accompanying this paper, the implementation in $\mathrm{R}$ is discussed in detail.

\subsection{A clinical trial in Schizophrenia}

A clinical trial was designed to compare the efficacy of risperidone versus haloperidol (active control) in the treatment of patients suffering from schizophrenia. The study included a total of $N=454$ patients that were treated for a period of eight weeks. Patients were assessed using two psychiatric rating scales, the Positive and Negative Syndrome Scale (PANSS; [19]) and the Brief Psychiatric Rating Scale (BPRS; [20]). PANSS is a 30 items scale that provides an operationalized, drug-sensitive instrument, which is highly useful for both typological 


\section{Statistics}

\section{in Medicine}

and dimensional assessment of schizophrenia. BPRS is basically a subscale of PANSS including only 18 items. The outcome of interest was the presence of a clinically relevant change in schizophrenic symptomatology as evaluated by the BPRS/PANSS scales. Clinically relevant change is defined as a reduction of $20 \%$ or more in the BPRS/PANSS scores, i.e, 20\% reduction in posttreatment scores relative to baseline scores.[21, 22]

Oftentimes in psychiatry, several instruments are available to assess a patient's mental condition and although there is not a clear gold standard among psychiatric rating scales, in the present study PANSS is the most complete and reliable instrument. Therefore, PANSS will be considered the main outcome or true endpoint and BPRS will be treated as the secondary outcome or potential surrogate endpoint. The main idea is to evaluate if a simpler and easier to administer scale like BPRS, could be reliably used as a substitute for a more complex scale, like PANSS, that requires more time and expertise to be administered.

\subsection{Results}

Two main analyses were carried out to evaluate BPRS as a surrogate for PANSS (we will loosely use BPRS and PANSS to refer to clinically relevant change in BPRS and clinically relevant change in PANSS, respectively). In the first analysis, the ICA and SPF were assessed using the two-step Monte Carlo procedure introduced in Alonso et al. [4] and Alonso et al. [5] and explained in Section 6. In the second analysis, the new MEP approach was first applied to calculate the maximum entropy relative frequencies associated with the distribution of the vector of potential outcomes and, afterward, the ICA and SPF were determined using these values.

Assessing the individual causal association Four settings were considered in the analysis regarding monotonicity: i) Monotonicity holds for $T$, i.e., assuming $P(\Delta T<0)=0$, ii) Monotonicity holds for $S$, i.e., assuming $P(\Delta S<0)=0$, iii) Monotonicity holds for $S$ and $T$, and iv) Monotonicity holds neither for $S$ nor for $T$. Each of the previous settings defined a region in $\Gamma_{D}$ where the Monte Carlo procedure was applied to study the behavior of $R_{H}^{2}$.

Undoubtedly, the results lead to rather uncertain conclusions regarding the validity of the surrogate. In fact, Figure 1 (left panel) displays the frequency distributions of $R_{H}^{2}$ for the different settings in the sensitivity analysis. The plots indicate that $R_{H}^{2}$ tends to take larger values in the region where monotonicity does not hold than in the other regions of $\Gamma_{D}$. Actually, the monotonicity assumption has a major impact on the results, e.g., the mean $R_{H}^{2}=0.54$ assuming no monotonicity is more than 4 times larger than the mean $R_{H}^{2}=0.13$ assuming monotonicity for both $S$ and $T$. The figure also shows that the ICA can take values ranging from about 0.0 to 0.8 . There are scenarios where both individual causal treatment effects are essentially independent $\left(R_{H}^{2} \approx 0\right)$ and others where the association is moderately high $\left(R_{H}^{2} \geq 0.79\right)$. When the procedure introduced in Section 6 was applied the following bounds for the ICA were obtained: i) Monotonicity holds for $T$ bounds equal [0.0; 0.62], ii) Monotonicity holds for $S$ bounds equal [0.01; 0.69], iii) Monotonicity holds for $S$ and $T$ bounds equal [0.0; 0.65], and iv) Monotonicity holds neither for $S$ nor for $T$ bounds equal [0.05; 0.77]. Globally, when all settings are combined, wider bounds are obtained $[0.0 ; 0.77]$. The analysis of the bounds again indicates a large level of uncertainty due to unidentifiability.

Maximizing the unconstrained dual convex programming problem, introduced in Section 4.1, led to the maximum entropy frequency distribution displayed in Table 4 . The MEP distribution has maximum entropy $\left(H_{\max }=1.9820\right)$ among all distributions in $\Omega_{C}$ and, consequently, it imposes the minimal structural constraints beyond those dictated by the data. In other words, from all the distributions in $\Omega_{C}, p^{*}$ is the one with minimal informational content. The MEP-ICA, i.e., the ICA obtained from $\boldsymbol{p}^{*}$ equals 0.492 (see Figure 1 left panel, vertical line). In addition, 1, 000 samples were drawn with replacement from the schizophrenia data set and the MEP-ICA was computed for each of them. The $2.5 \%$ and $97.5 \%$ quantiles of the bootstrapped distribution equaled 0.414 and 0.587 , respectively. The same quantiles of the bootstrapped maximum entropy distribution (vector $\mathbf{p}^{*}$ ) were computed and are shown in Table 4. 


\section{Statistics}

The results based on the MEP are a valuable addition to the sensitivity analysis and offer a new framework for its interpretation. As it can be learned from Figure 1 (left panel), although assuming no monotonicity leads to ICA values that are mostly larger than the MEP-ICA, values smaller than the MEP-ICA have also some mass in this setting. However, assuming monotonicity for one or both endpoints leads to frequency distributions for the ICA that are almost completely to the left of the MEP-ICA. Interestingly, the unverifiable monotonicity assumptions tend to move the ICA values to the left and to right of the MEP-ICA, i.e., the value obtained from the most noninformative analysis, with more extreme deviations observed when monotonicity is assumed for both endpoints. Additionally, although both weak and moderately high associations are compatible with the data at hand, the MEP analysis shows that the assessment based on the distribution with minimal informational content, only indicates a moderate association between the individual causal effects. Hence, one may argue that a moderate association may be a reasonable framework for the decision making process. In spite of its appealing interpretation, the MEP approach should basically be used as an addition to the sensitivity analysis and, therefore, the results emanating from it should not be over-interpreted or considered the ultimate truth but being discussed in the broader scientific framework.

\section{[Insert Table 4 about here]}

[Insert Figure 1 about here]

The maximum entropy frequency distribution displayed in Table 4 makes clear statements about reality. Although untestable from the data, these implications of the MEP distribution could be discussed with clinicians to assess their plausibility. For example, $p_{0000}^{*}=0.19$ implies that about $20 \%$ of the patients respond neither on $T$ nor on $S$, irrespective of the treatment received (i.e., $T_{0}=T_{1}=S_{0}=S_{1}=0$; never-responders), whereas $p_{1111}^{*}=0.21$ implies that about $20 \%$ of the patients are always-responders (i.e., $T_{0}=T_{1}=S_{0}=S_{1}=1$ ). Similarly, $p_{0101}^{*}=$ 0.2402 implies that about $25 \%$ of the patients have positive responses on $T$ and $S$ under the experimental treatment but not under the control treatment (i.e., $T_{1}=S_{1}=1$ and $T_{0}=S_{0}=0$ ), and so on.

The use of the ECT in this specific case study implies that $95 \%$ of all $\boldsymbol{p} \in \Omega_{C}$ satisfy $1.9647 \leq H(\boldsymbol{p}) \leq 1.9818$ and, hence, the distributions compatible with the data do tend to highly concentrate around the maximum entropy configuration.

One may speculate that, although the association between the potential outcomes for the true $\left(T_{0}, T_{1}\right)$ and surrogate $\left(S_{0}, S_{1}\right)$ endpoints are not identifiable from the data, given the similarities between the therapies in the study, patients who would do well on one treatment are likely to do well on the other and, therefore, these potential outcomes are plausibly positively associated. These type of untestable assumptions can easily be incorporated into the Monte Carlo procedure by considering in the analysis only those sampled $\pi$ vectors that produce an association between the potential outcomes larger than or equal to a prespecified value. The right panel of Figure 1 shows the frequency distributions for the ICA obtained when $R_{H}^{2}\left(T_{0}, T_{1}\right)>c$ and $R_{H}^{2}\left(S_{0}, S_{1}\right)>c$ with $c$ equal to 0.25 and 0.45 . In order to keep the length of the manuscript at a reasonable level, we present here only the results obtained under the no monotonicity assumption but the interested reader can find the complete analysis in the Supplementary Materials Part B. Clearly, in the region of $\Gamma_{D}$ where the association is stronger $(c=0.45)$ low ICA values are more frequent than in the region where the association is weaker $(c=0.25)$. These results seem to be in agreement with the results obtained in the general sensitivity analysis, where lower values for the ICA were more frequently found in the regions where monotonicity held. Indeed, assuming monotonicity for one endpoint induces a positive association between the potential outcomes for that endpoint. For instance, assuming monotonicity for the surrogate implies that the odds ratio quantifying the association between the potential outcomes for the surrogate equals infinity. 


\section{Statistics}

\section{in Medicine}

Assessing the Surrogate Predictive Function In the following, the results of the analysis based on the SPF, under the no monotonicity assumption, are presented. The interested reader can find the complete analysis and discussion in the supplementary materials. Figure 2 shows the behavior of the SPF on $\Gamma_{D}$ (grey histograms). The bottom left figure suggests that the probability of a false positive result may be rather small, with mean $r(-1,1)=P(\Delta T=-1 \mid \Delta S=1)=0.0394$ and range $[0.0001 ; 0.3263]$. In addition, the top right figure suggests that the probability of a false negative result may be small as well, with mean $r(1,-1)=P(\Delta T=1 \mid \Delta S=-1)=$ 0.0468 , but now the range for this probability is much wider $[0.0001 ; 0.8288]$, hinting on a substantial level of uncertainty due to the unidentifiability of this parameter. Further, the top left figure shows that, although a negative individual causal treatment effect on the BPRS may typically lead to a negative individual causal treatment effect on the PANSS, with mean $r(-1,-1)=P(\Delta T=-1 \mid \Delta S=-1)=0.7815$, a large level of uncertainty is also present in this scenario (range between 0.0009 and 0.9953 ).

[Insert Figure 2 about here]

The histogram in the center figure offers some degree of support for causal necessity with mean $r(0,0)=$ $P(\Delta T=0 \mid \Delta S=0)=0.8339$. Thus, a lack of individual causal treatment effect on the BPRS (i.e., $\Delta S=0$ ) may be indicative of a lack of individual causal treatment effect on PANSS (i.e., $\Delta T=0$ ). However, there is a substantial uncertainty in this scenario as well, with $P(\Delta T=0 \mid \Delta S=0)$ taking values as small as 0.0798 and as large as 0.9905 for some points in $\Gamma_{D}$.

Overall, there is some evidence that $r(i, i)=P(\Delta T=i \mid \Delta S=i)$ may be relatively large for all $i$ (the main diagonal in the figure), with all means $\geq 0.7815$, all medians $\geq 0.8470$ and all modes $\geq 0.8900$. However, as the previous discussion indicates, the lack of indentifiability introduces a substantial level of uncertainty regarding the true value of these probabilities.

\section{[Insert Table 5 about here]}

Table 5 shows the MEP-based SPF estimates and, for comparison purposes, their values are also given in Figure 2 using vertical black lines. Overall, the MEP-based $r(i, j)$ were of the same order of magnitude as the sensitivityanalysis-based mean and median $r(i, j)$ values (see also Table 5). However, in some situations large differences were observed, for instance, for $r(-1,1)$ the MEP analysis led to an estimate that is four and three times smaller than the sensitivity-analysis-based mean and median, respectively. Here again the MEP results offer a useful framework for the interpretation of the sensitivity analysis. In fact, let us consider, for example, $r(-1,-1)$, for this probability the mean of the sensitivity analysis equaled 0.7815 but the range of values obtained for it shows a huge level of uncertainty due to the unidentifiability. Actually, $r(-1,-1)$ can take values as small as 0.00 and as large as 0.99 for different points in $\Gamma_{D}$, i.e., basically it can cover the entire $(0,1)$ interval. Although all these values are equally compatible with the data at hand, the MEP analysis led to $r(-1,-1)=0.8300$ and, therefore, the most general assessment, i.e., the assessment based on the most non-informative distribution compatible with the data, indicates a rather large value for this probability. If no additional information is available, then it would be reasonable to consider only a large value of $r(-1,-1)$ as a reference in the decision making process. Here again clinical consideration should play an important role in the decision making process.

\section{Conclusions}

In the present work, a MEP approach was introduced for the evaluation of a binary outcome as a putative surrogate for a binary true endpoint within a causal inference framework. The method produces results that have a simple, 
yet appealing interpretation in terms of informational content. Philosophically, the MEP is related to Laplace's principle of indifference, sometimes called the principle of insufficient reason, which basically states that if $n$ possibilities are indistinguishable except for their names, then each possibility should be assigned a probability equal to $1 / n$. The MEP also underlies the theory of non-informative priors prevalent in Bayesian theory.

In spite of its appealing interpretation, we strongly advise to use the MEP approach, or any other identifying method for that matter, as part of a sensitivity analysis and not as a stand alone procedure. We strongly believe that, when facing a non-identifiability problem, quantitative methods should be seen as a framework for a wider scientific debate and their results should be interpreted with extreme caution.

Another important research line is the incorporation of subject-specific information when calculating the maximum entropy distribution or in order to obtain narrower bounds for the ICA and SPF. Using subject-specific information has the potential to produce more precise conclusions and this topic will be the focus of future research.

Ultimately, a thoughtful discussion that takes into account the results obtained from different approaches (sensitivity analysis, bounds for the effects of interest, point estimates based on different identifiability conditions, etc.), clinical considerations and practical issues, will be necessary before a final decision can be made on the use of a surrogate endpoint.

\section{Supplementary Material}

A Web Appendix that details the analyses of the case study (using the R package Surrogate and that provides a proof for Lemma 2 is available at the website of Statistics in Medicine. 


\section{Statistics}

\section{in Medicine}

\section{References}

[1] Joffe, M. M., and Greene, T. (2009). Related causal frameworks for surrogate outcomes. Biometrics, 65, 530538.

[2] Gilbert, P. B., and Hudgens, M. G. (2008), Evaluating candidate principal surrogate endpoints. Biometrics, 64, $1146-1154$.

[3] Li, Y., Taylor, J. M. G., and Elliott, M. R. (2010). A Bayesian approach to surrogacy assessment using principal stratification in clinical trials. Biometrics, 58, 21-29.

[4] Alonso A., Van der Elst W., Molenberghs G., Buyse M., and Burzykowski T. (2016), A causal-inference approach for the validation of surrogate endpoints based on information theory and sensitivity analysis. Biometrics 72, 669-677.

[5] Alonso A., and Van der Elst W. (2016), Assessing a surrogate predictive value: A causal inference approach. Statistics in Medicine 36, 1083-1098.

[6] Bernardo, Jose M. (1979). Reference posterior distributions for Bayesian inference. Journal of the Royal Statistical Society, Series B, 41 (2), 113-147.

[7] Berger J, Bernardo J, Dongchu S (2009). The Formal Definition of Reference Priors. Annals of Statistics, 37(2), 905-938.

[8] Jaynes, E. T. (1985), Where do we go from here? In C. R. Smith and J. W. T. Grandy, editors, MaximumEntropy and Bayesian Methods in Inverse Problems, Kluwer Academic Publishers, 21-58.

[9] Gadbury L G, Iyer H K and Jeffrey M A (2004). Individual treatment effects in randomized trials with binary outcomes. Journal of Statistical Planning and Inference, 121, 163-174.

[10] Rubin, D. B. (1980). Randomization analysis of experimental-data the Fisher randomziation test? Comment. Journal of the American Statistical Association, 75, 591-593.

[11] Holland, P. W. (1986). Statistics and Causal Inference. Journal of the American Statistical Association, 81, 945-960.

[12] Plackett, R. L. (1965). A class of bivariate distributions. Journal of the American Statistical Association, 60, 516-522.

[13] Shannon, C. (1948). A mathematical theory of communication. Bell System Technical Journal, 27, 379-423 and 623-656.

[14] Elliott, M. R., Li, Y., and Taylor, J. M. G. (2013). Accommodating missingness when assessing surrogacy via principal stratification. Clinical Trials, 10, 363-377.

[15] Alonso, A., Van der Elst, W., Molenberghs, G., Buyse, M., and Burzykowski, T. (2015). On the relationship between the causal-inference and meta-analytic paradigms for the validation of surrogate endpoints. Biometrics, 70, $15-24$.

[16] Frangakis C. E., and Rubin, D. B. (2002). Principal stratification in causal inference. Biometrics, 58, 21-29. 
[17] Edwin, T. J. (1982). On the rationale of maximum-entropy methods. Proceedings of the IEEE, 70, 939-952.

[18] Brockett, P. L., Charnes, A., and Cooper, W. W. (1980), MDI estimation via unconstrained convex programming. Communications in Statistics, 9, 223-234.

[19] Singh, M., and Kay, S. (1975), A comparative study of haloperidol and chlorpromazine in terms of clinical effects and therapeutic reversal with benztropine in schizophrenia. Theorectical implications for potency differences among neuroleptics. Psychopharmacologia, 43, 103-113.

[20] Overall, J., and Gorham, D. (1962). The Brief Psychiatric Rating Scale. Psychological Reports, 10, 799-812.

[21] Kane, J., Honigfeld, G., Singer, J., and Meltzer, H. (1988). Clozapine for the treatment-resistant schizophrenic. A double-blind comparison with chlorpromazine. Archives of General Psychiatry, 45, 789-796.

[22] Leucht, S., Kane, J. M., Kissling, W., Hamann, J., Etschel, E., and Engel, R. (2005). Clinical implications of the Brief Psychiatric Rating Scale Scores. British Journal of Psychiatry, 187, 366-371. 


\section{Statistics}

\section{in Medicine}

Table 1. Definition of abbreviations used in the manuscript.

\begin{tabular}{|c|l|}
\hline Abbreviation & \multicolumn{1}{c|}{ Definition } \\
\hline ICA & $\begin{array}{l}\text { Individual Causal Association defined as the association between the individual causal treatment } \\
\text { effects on the surrogate and true endpoints }\end{array}$ \\
\hline MEP & $\begin{array}{l}\text { Maximum Entropy Principle. It states that, when making inferences based on incomplete } \\
\text { information, one should use the probability distribution that has the maximum entropy permitted } \\
\text { by the data at hand. }\end{array}$ \\
\hline ML(E) & Maximum Likelihood (Estimator) \\
\hline SPF & $\begin{array}{l}\text { Surrogate Predictive Function defined as the probability that the individual causal treatment } \\
\text { effect on the true endpoint will take certain value given a value of individual causal treatment } \\
\text { effect on the surrogate }\end{array}$ \\
\hline ETC & Entropy Concentration Theorem introduce by Edwin [17] \\
\hline
\end{tabular}


Table 2. Distribution of $\boldsymbol{\Delta}=(\Delta T, \Delta S)^{\prime}$.

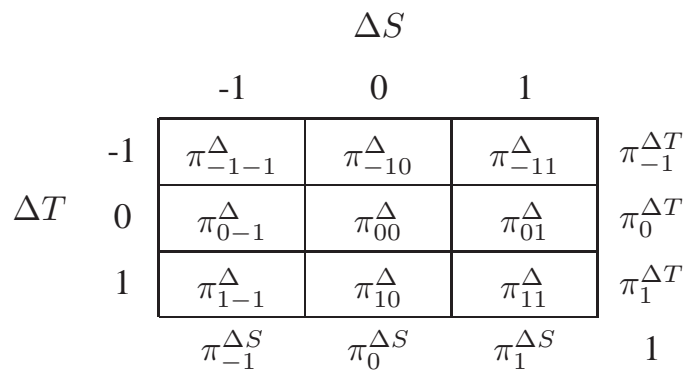




\section{Statistics}

Table 3. Summary of simulation results. Data generated assuming monotonicity for $S$ and $T$, and analysed under the four different montonicity scenarios.

\begin{tabular}{ccccc}
\hline \multicolumn{5}{c}{ Data generated assuming monotonicity for S and T } \\
\hline True ICA & $\begin{array}{c}\text { Mono. } \\
\text { Assump. }\end{array}$ & $\begin{array}{c}\text { \% True ICA } \\
\text { included }\end{array}$ & $\begin{array}{c}\text { Mean } \\
\text { min(ICA) }\end{array}$ & $\begin{array}{c}\text { Mean } \\
\max (\text { ICA })\end{array}$ \\
\hline$(0,0.1]$ & No & 0.385 & 0.044 & 0.257 \\
$(0.1,0.2]$ & No & 0.754 & 0.049 & 0.282 \\
$(0.2,0.3]$ & No & 0.611 & 0.058 & 0.313 \\
$(0.3,0.4]$ & No & 0.434 & 0.072 & 0.328 \\
$(0.4,0.5]$ & No & 0.338 & 0.088 & 0.370 \\
\hline$(0,0.1]$ & S & 0.901 & 0.003 & 0.458 \\
$(0.1,0.2]$ & S & 0.987 & 0.006 & 0.478 \\
$(0.2,0.3]$ & S & 0.970 & 0.011 & 0.522 \\
$(0.3,0.4]$ & S & 0.921 & 0.022 & 0.552 \\
$(0.4,0.5]$ & S & 0.833 & 0.036 & 0.591 \\
\hline$(0,0.1]$ & T & 0.909 & 0.002 & 0.464 \\
$(0.1,0.2]$ & T & 0.994 & 0.005 & 0.488 \\
$(0.2,0.3]$ & T & 0.970 & 0.010 & 0.529 \\
$(0.3,0.4]$ & T & 0.920 & 0.019 & 0.551 \\
$(0.4,0.5]$ & T & 0.879 & 0.035 & 0.596 \\
\hline$(0,0.1]$ & S and T & 0.999 & 0.000 & 0.413 \\
$(0.1,0.2]$ & S and T & 0.999 & 0.002 & 0.427 \\
$(0.2,0.3]$ & S and T & 0.997 & 0.007 & 0.471 \\
$(0.3,0.4]$ & S and T & 1.000 & 0.014 & 0.538 \\
$(0.4,0.5]$ & S and T & 1.000 & 0.031 & 0.617 \\
\hline
\end{tabular}

* Monotonicity assumption made for the analysis

** Percentage of cases in which the true ICA was included in [min., max.] 
Table 4. Distribution of $\boldsymbol{Y}$ (right hand columns contain bootstrap $95 \%$ standard deviations and confidence intervals)

\begin{tabular}{cccc|c|ccc}
\hline$T_{0}$ & $T_{1}$ & $S_{0}$ & $S_{1}$ & $\boldsymbol{\pi}$ & $\boldsymbol{p}^{*}$ & $S D$ & $95 \% C I$ \\
\hline 0 & 0 & 0 & 0 & $\pi_{0000}$ & 0.195 & 0.021 & {$[0.16 ; 0.24]$} \\
0 & 1 & 0 & 0 & $\pi_{0100}$ & 0.013 & 0.005 & {$[0.00 ; 0.03]$} \\
0 & 0 & 1 & 0 & $\pi_{0010}$ & 0.022 & 0.01 & {$[0.01 ; 0.04]$} \\
0 & 0 & 0 & 1 & $\pi_{0001}$ & 0.023 & 0.01 & {$[0.01 ; 0.04]$} \\
0 & 1 & 0 & 1 & $\pi_{0101}$ & 0.240 & 0.02 & {$[0.20 ; 0.29]$} \\
1 & 0 & 0 & 0 & $\pi_{1000}$ & 0.021 & 0.01 & {$[0.01 ; 0.04]$} \\
1 & 0 & 1 & 0 & $\pi_{1010}$ & 0.174 & 0.02 & {$[0.14 ; 0.22]$} \\
1 & 0 & 0 & 1 & $\pi_{1001}$ & 0.002 & 0.01 & {$[0.00 ; 0.01]$} \\
1 & 1 & 1 & 0 & $\pi_{1110}$ & 0.011 & 0.01 & {$[0.00 ; 0.01]$} \\
1 & 1 & 0 & 1 & $\pi_{1101}$ & 0.026 & 0.01 & {$[0.00 ; 0.02]$} \\
1 & 0 & 1 & 1 & $\pi_{1011}$ & 0.020 & 0.01 & {$[0.01 ; 0.03]$} \\
1 & 1 & 1 & 1 & $\pi_{1111}$ & 0.215 & 0.02 & {$[0.17 ; 0.26]$} \\
0 & 1 & 1 & 0 & $\pi_{0110}$ & 0.002 & 0.01 & {$[0.00 ; 0.01]$} \\
0 & 0 & 1 & 1 & $\pi_{0011}$ & 0.003 & 0.01 & {$[0.00 ; 0.01]$} \\
0 & 1 & 1 & 1 & $\pi_{0111}$ & 0.027 & 0.01 & {$[0.01 ; 0.04]$} \\
1 & 1 & 0 & 0 & $\pi_{1100}$ & 0.001 & 0.01 & {$[0.00 ; 0.01]$} \\
\hline \multicolumn{7}{c}{}
\end{tabular}




\section{Statistics}

Table 5. Summary descriptives of the sensitivity-based $r(i, j)$ (left columns) and the MEP-based results (right columns, bootstrap standard deviations and confidence intervals are provided).

\begin{tabular}{lcccccc}
\hline & \multicolumn{2}{c}{ Sensitivity-based } & & \multicolumn{3}{c}{ MEP-based } \\
\cline { 2 - 3 } \cline { 5 - 7 } & Mean & Median & & point estimate & SD & 95\% CI \\
\cline { 2 - 3 }$r(-1,-1)$ & 0.7815 & 0.8470 & & 0.8300 & 0.0360 & {$[0.75 ; 0.89]$} \\
$r(0,-1)$ & 0.1717 & 0.1199 & & 0.1627 & 0.0329 & {$[0.10 ; 0.23]$} \\
$r(1,-1)$ & 0.0468 & 0.0270 & & 0.0072 & 0.0035 & {$[0.00 ; 0.02]$} \\
$r(-1,0)$ & 0.0894 & 0.0661 & & 0.0838 & 0.0170 & {$[0.05 ; 0.12]$} \\
$r(0,0)$ & 0.8339 & 0.8764 & & 0.8340 & 0.0215 & {$[0.79 ; 0.88]$} \\
$r(1,0)$ & 0.0767 & 0.0554 & & 0.0822 & 0.0186 & {$[0.05 ; 0.12]$} \\
$r(-1,1)$ & 0.0394 & 0.0288 & & 0.0085 & 0.0035 & {$[0.00 ; 0.02]$} \\
$r(0,1)$ & 0.1258 & 0.1043 & & 0.1678 & 0.0301 & {$[0.11 ; 0.23]$} \\
$r(1,1)$ & 0.8348 & 0.8597 & & 0.8237 & 0.0335 & {$[0.76 ; 0.89]$} \\
\hline
\end{tabular}




\section{Statistics}
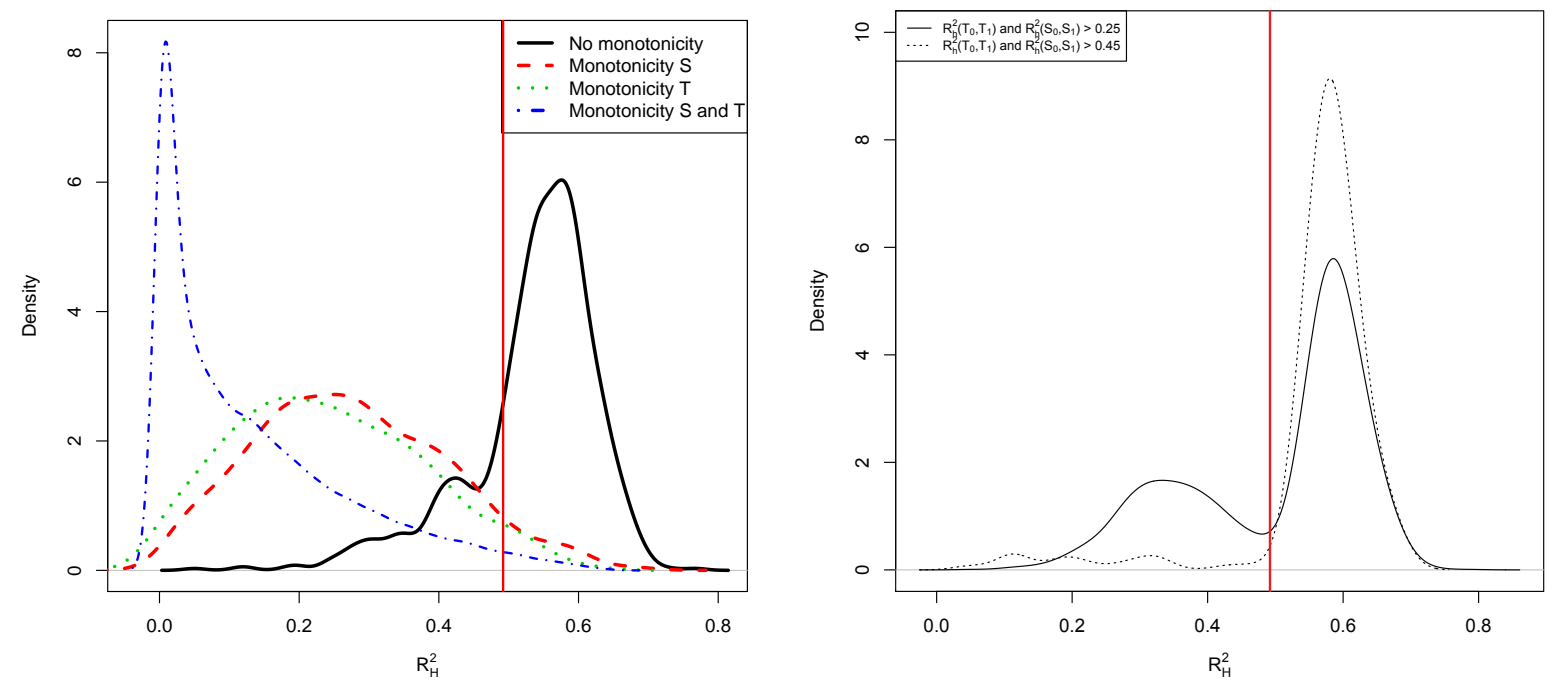

Figure 1. Frequency densities for the ICA and MEP-based ICA value (vertical line). Left: General sensitivity analysis. Right: Sensitivity analysis fixing the association between the potential outcomes and assuming no monotonicity 


\section{Statistics}
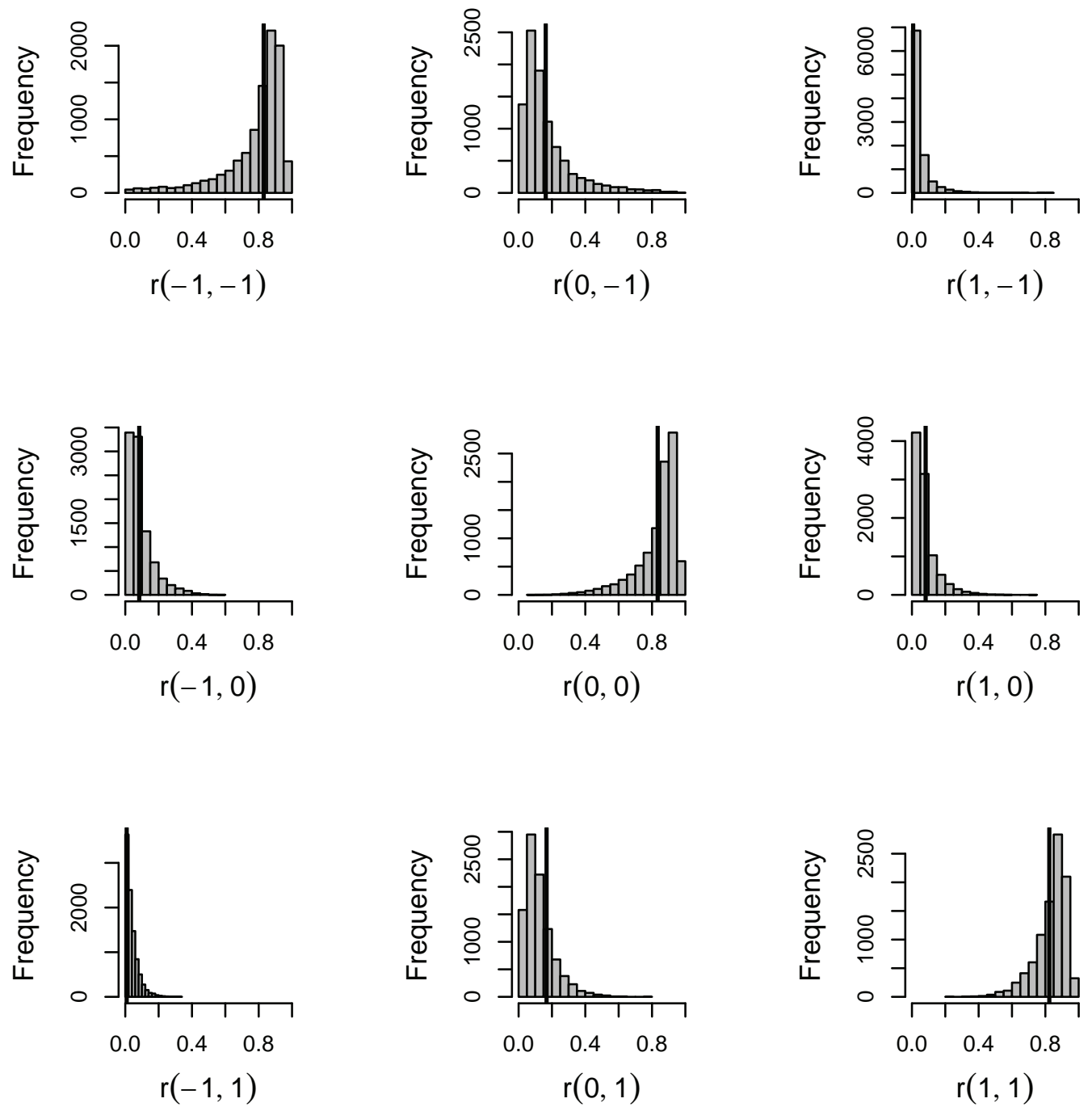

Figure 2. Surrogate predictive function (grey histograms) and MEP-based SPF (vertical black lines) 